\title{
Dietary Fat Intake and the Risk of Incident Dementia in the Rotterdam Study
}

\author{
Sandra Kalmijn, MD, ${ }^{*} \dagger \ddagger$ Lenore J. Launer, PhD, ${ }^{* \dagger}$ Alewijn Ott, MD, ${ }^{*}$ Jacqueline C. M. Witteman, PhD, ${ }^{*}$ \\ Albert Hofman, MD,* and Monique M. B. Breteler, MD*
}

\begin{abstract}
A high intake of saturated fat and cholesterol and a low intake of polyunsaturated fatty acids have been related to an increased risk of cardiovascular disease. Cardiovascular disease has been associated with dementia. We investigated the association between fat intake and incident dementia among participants, age 55 years or older, from the populationbased prospective Rotterdam Study. Food intake of 5,386 nondemented participants was assessed at baseline with a semiquantitative food-frequency questionnaire. At baseline and after an average of 2.1 years of follow-up, we screened for dementia with a three-step protocol that included a clinical examination. The risk of dementia at follow-up (RR [ $95 \%$ CI]) was assessed with logistic regression. After adjustment for age, sex, education, and energy intake, high intakes of the following nutrients were associated with an increased risk of dementia: total fat $(R R=2.4[1.1-5.2])$, saturated fat $(R R=1.9[0.9-4.0])$, and cholesterol $(R R=1.7[0.9-3.2])$. Dementia with a vascular component was most strongly related to total fat and saturated fat. Fish consumption, an important source of $n-3$ polyunsaturated fatty acids, was inversely related to incident dementia $(R R=0.4[0.2-0.9])$, and in particular to Alzheimer's disease $(R R=0.3[0.1-$ 0.9]). This study suggests that a high saturated fat and cholesterol intake increases the risk of dementia, whereas fish consumption may decrease this risk.
\end{abstract}

Kalmijn S, Launer LJ, Ott A, Witteman JCM, Hofman A, Breteler MMB. Dietary fat intake and the risk of incident dementia in the Rotterdam study. Ann Neurol 1997;42:776-782

As the population includes a growing proportion of older people, dementia will become an increasing burden to society and the individual. Therefore, research on potentially modifiable risk factors, such as diet, is of enormous relevance. Few studies have reported on the relation between diet and dementia [1-3]. Because the study designs were cross-sectional and the demented subjects were usually nursing-home residents, it was difficult to ascertain whether their diet was the cause or consequence of dementia.

In this study, we focused on different components of fat intake, including total fat, saturated fat, cholesterol, and polyunsaturated fatty acids (PUFAs). Saturated fat and cholesterol may increase the risk of dementia, because they are associated with adverse cardiovascular events $[4-6]$. These events, in turn, have been related to both vascular dementia and Alzheimer's disease [7-10]. On the other hand, n-3 PUFAs, which have antithrombotic effects [11], and n-6 PUFAs, which have a beneficial effect on the lipid profile $[12,13]$, may reduce the risk of dementia. We examined the association between fat intake and (subtypes of) dementia in a prospective population-based study, in which dietary data were collected at baseline before the onset of dementia.

\section{Subjects and Methods \\ Study Population}

We used data from the Rotterdam Study, which is a singlecenter prospective population-based study, designed to investigate determinants of selected chronic diseases and disabilities in older persons [14]. The conduct of the study was approved by the Medical Ethics Committee of the Erasmus University and written consent was obtained from all participants. All residents of a suburb in Rotterdam, age 55 years and older, were invited and of the 10,275 eligible subjects, $7,983(78 \%)$ agreed to participate. During a home visit, trained interviewers administered a questionnaire, covering, among other areas, sociodemographic background, medical history, and medication use. This was followed by two clinical examinations at the research center. Subjects living in nursing homes were visited at home. The food-frequency questionnaire was included in the baseline examinations after the pilot phase, and offered to independently living subjects, who underwent additional clinical examinations $(\mathrm{n}=$ 6,250). As part of the protocol, subjects who scored below 80 points on the cognitive portion of the Cambridge Mental
From the ${ }^{*}$ Department of Epidemiology and Biostatistics, Erasmus University Medical School, and ₹Netherlands Institute for Health Sciences, Rotterdam; and $†$ Department of Chronic Diseases and Environmental Epidemiology, National Institute of Public Health and the Environment, Bilthoven, The Netherlands.
Received May 14, 1997, and in revised form Jul 25. Accepted for publication Jul 30, 1997.

Address correspondence to Dr Kalmijn, Department of Epidemiology and Biostatistics, Erasmus University Rotterdam, PO Box 1738 , 3000 DR Rotterdam, The Netherlands. 
Disorders of the Elderly Examination (CAMDEX) [15] ( $\mathrm{n}=$ 122), which is the neuropsychological test administered in the case-finding procedure for dementia, were excluded because they were thought to be unable to provide reliable answers regarding their food patterns. Furthermore, 482 unselected subjects did not receive a dietary questionnaire due to logistic reasons, and 212 respondents were excluded from the sample because of logical inconsistencies in the dietary interviews, resulting in 5,434 completed questionnaires. Baseline data were collected from May 1990 to June 1993. Follow-up data were obtained until December 1994. Incident dementia was ascertained in two ways, ie, on the basis of a study examination in those attending the follow-up visit, or from medical records if the subject refused reexamination or had died before reexamination.

\section{Dietary Intake}

Dietary intake was assessed with a 170-item semiquantitative food-frequency questionnaire, which is a modified version of a reliable and valid questionnaire $[16,17]$. Modifications of the original questionnaire included a different mode of administration and the addition of more detailed questions on vegetable, fruit, and meat consumption. The questionnaire was administered in two stages. In the first stage, respondents were asked to report foods consumed regularly (at least twice a month) in the last year. This took place at home. The second stage was completed during the second visit to the research center. After a review of the checked food items, a trained dietician asked the respondent how much and how often each food item was consumed. The whole procedure was completed in 40 minutes. Average daily nutrient intake was calculated by multiplying the frequency and amount consumed for each food item by its nutrient content listed in an automated version of the Dutch Food Composition Table [18]. We used linoleic acid intake as a marker of n-6 PUFA intake, because it is the most important n-6 PUFA in Western populations [13]. The intake of the $n-3$ PUFAs eicosapentaenoic acid and docosahexaenoic acid was estimated by fish consumption, because fish is their main source.

\section{Dementia Case Finding}

Case-finding procedures for dementia have been described elsewhere [19]. As in the baseline examination, the follow-up study examination consisted of a three-stage procedure. First, all participants were screened with the 30-point Mini-Mental State Examination (MMSE) [20] and the short version of the Geriatric Mental State Schedule (GMS-A, organic level) [21]. Subjects with an MMSE score below 26, or a GMS score greater than 0 were considered screen positive. They were subsequently examined with the CAMDEX, which included an informant interview [15]. Participants who were judged to be demented or suspected of dementia after the CAMDEX were examined by a neurologist and tested by a neuropsychologist; a part also underwent magnetic resonance imaging of the brain. Additional information for those who did not complete the diagnostic phase was obtained from the general practitioner, the regional institute for outpatient mental health care, and specialist medical files. Diagnosis of Alzheimer's disease $(A D)$ was based on National Institute of Neurological and Communicative Disorders and Stroke-
Alzheimer's Disease and Related Disorders Association (NINCDS-ADRDA) criteria [22]. Patients with this diagnosis had a gradually progressive global decline of cognitive functions for which no medical cause was found. Dementia patients with a history of stroke, who otherwise fulfilled $A D$ criteria, were subclassified as having $\mathrm{AD}$ with cerebrovascular disease. Vascular dementia was diagnosed in accordance with National Institute of Neurological Disorders and Strokeand Association Internationale pour la Recherche et l'Enseignement en Neurosciences (NINDS-AIREN) criteria [23]. In these patients the onset of dementia was related to cerebrovascular disease. They generally had an acute onser of dementia symptoms with stepwise progression of the disease. Diagnostic and Statistical Manual of Mental Disorders, third edition, revised (DSM-III-R) criteria were used to classify other dementias [24].

\section{Other Measurements}

The following variables were considered as possible confounding variables: age, sex, total energy intake, cigarette smoking (current, former, or never), alcohol consumption (none, $<1$ drink $[13.2 \mathrm{~g}], 1-2$ drinks, or $\geq 3$ drinks per day), fiber consumption, antioxidant intake, and level of education (completed primary education; lower vocational training or general education; intermediate vocational training or intermediate and higher general education; and higher vocational training, college, or university) (United Nations Educational, Scientific and Cultural Organization, UNESCO).

A history of cardiovascular disease at baseline, indicators of atherosclerosis, and total and high-density lipoprotein cholesterol were considered as possible mediators of the association between dietary factors and dementia. Data on medical history were obtained at the home interview. A history of myocardial infarction was considered present if the subject reported an event that was diagnosed by a physician and led to hospitalization. A history of stroke was considered present if a self-reported event was confirmed by either a detailed history, neuroimaging, or discharge reports collected from the general practitioner or neurologist [25]. The following indicators of atherosclerosis were used: plaques in the carotid arteries, intima-media wall thickness of the carotid artery, and ankle-brachial index of systolic blood pressure. Measurement methods are extensively described elsewhere [26]. Wall thickness was measured in a relatively small but random subset of subjects $(n=1,935)$, and we restricted the analyses on atherosclerosis to this subset.

\section{Statistical Analysis}

Complete information on diet, incidence of dementia, and confounding factors was available for 5,386 respondents. Mean daily nutrient intake in incident demented and nondemented subjects, and the difference $(95 \%$ confidence intervals [CIs]) between the two groups, was obtained from analysis of covariance, controlling for age. Because of the high correlation between fat and total energy intake, energyadjusted fat and fish intakes were calculated with the residual method [27]. This method gives an estimation of the regression coefficient, which is independent of total energy intake. Energy-adjusted intake of, for example, saturated fat was cal- 
culated as the summation of the residuals from regressing saturated fat on total energy intake and a constant equal to the expected saturated fat intake at the level of the mean total energy intake. Energy-adjusted intakes of the nutrients of interest were grouped into tertiles, with the lowest tertile as the reference group.

We used multiple logistic regression analysis to estimate the relative risks ( $R R s$ ) for the associations between fat intake, fish consumption, and incident dementia. We included confounding variables in the model. The $p$ value for linear trend was assessed by putting the categorical variable as a continuous variable in the model. We also investigated whether there was effect modification by sex or age by including the product terms of the nutrients with sex or age in the model.

\section{Results}

Baseline characteristics are given in Table 1. Fifty-eight (1.1\%) of the 5,386 subjects became demented during follow-up (mean, 2.1 years $[\mathrm{SD}=0.8]$ ); $42(72 \%)$ were classified as having $\mathrm{AD}$ (including 5 with $\mathrm{AD}$ and cerebrovascular disease), 7 (12\%) as having vascular dementia, and $9(16 \%)$ as having orher types of dementia. Age-adjusted mean intake of total and saturated fat was higher, and fish consumption lower, in subjects with incident dementia (Table 2).

Subjects in the highest tertiles of total fat, saturated far, and cholesterol intake had an increased risk of dementia $(R R=2.4,95 \%$ CI: $1.1-5.2 ; R R=1.9,95 \%$ CI: $0.9-4.0$; and $R R=1.7,95 \%$ CI: $0.9-3.2$, respectively) (Table 3). High total and saturated fat intakes were most strongly associated with an increased risk of dementia with a vascular component (vascular dementia and $\mathrm{AD}$ with cerebrovascular disease). Fish consumption was associated with a reduced risk of dementia $(R R=0.4,95 \% \mathrm{CI}: 0.2-0.9)$ and especially of $\mathrm{AD}$ without cerebrovascular disease (Table 4). Linoleic acid was also associated with a lower risk of dementia, al-

Table 1. Baseline Characteristics of Participants Who Completed the Food-Frequency Questionnaire $(n=5,386)$ : The Rotterdam Study

Characteristics

Mean age (yr)

$67.7(7.8)^{\mathrm{a}}$

Sex (men/women)

$2,204 / 3,182$

Primary education only

$1,871(34.7 \%)$

Median baseline MMSE score (range)

$28(16-30)$

Mean alcohol consumption, $\geq 1$ drink/day $1,518(28.2 \%)$

Current smokers ${ }^{\mathrm{b}}$

Former smokers ${ }^{\mathrm{b}}$

$1,256(23.4 \%)$

$2,305(42.9 \%)$

History of stroke

$97(1.8 \%)$

History of myocardial infarction ${ }^{\mathrm{d}}$

$380(7.1 \%)$

${ }^{\mathrm{a}}$ Standard deviation in parentheses.

${ }^{b}$ Information available for 5,377 subjects.

Information available for 5,290 subjects.

${ }^{\mathrm{d}}$ Information available for 5,321 subjects.

MMSE $=$ Mini-Mental State Examination. though not significantly $(R R=0.6,95 \%$ CI: $0.3-1.2)$ These associations did not change after additional adjustment for cigarette smoking, alcohol consumption, fiber consumption, antioxidant intake, or serum total and high-density lipoprotein cholesterol. Entering the indicators of atherosclerosis into the model did not change the associations, compared with the associations in the same subsample, but without atherosclerosis in the model (data not shown). Adjustment for stroke or myocardial infarction did not alter the results; neither did exclusion of subjects with a history of stroke or myocardial infarction or exclusion of subjects who were using a prescribed diet.

\section{Discussion}

This large follow-up study showed an association between dietary factors and incidence of dementia. High intakes of total fat, saturated fat, and cholesterol were related to an increased risk of dementia, and total and saturated fat in particular to dementia with a vascular component. Fish consumption was associated with a reduced risk of dementia, and most strongly with $\mathrm{AD}$. To our knowledge, this is the first prospective cohort study that investigated the association between fat intake and incident dementia.

Some methodological problems of this study must be discussed. Dietary data collected from subjects who are cognitively impaired may be less reliable, because they may underestimate or overestimate their dietary intake, leading to differential misclassification [28]. Subjects with dementia at follow-up may more often be cognitively impaired at baseline than those who were not demented at follow-up. It does not appear very likely, however, that they consistently overreported the consumption of foods with a high saturated fat and cholesterol content and underreported the consumption of fish. In addition, we attempted to reduce systematic reporting error by a priori excluding nursing-home residents, individuals with low baseline scores on the CAMDEX, and questionnaires with logical inconsistencies. This exclusion, on the other hand, increases the possibility of response bias. The incidence of dementia is more likely to have been higher in those who did not complete the dietary questionnaire, but it is not very likely that they also had a lower far intake and a higher fish consumption. Therefore, response bias is not a plausible explanation for these findings. Another methodological problem would occur when subjects with incident dementia altered their food pattern at baseline, because of behavioral changes in preclinical stages of dementia. In our sample, however, subjects with incident dementia performed relatively well at the baseline cognitive screening tests. Still, we cannot fully exclude the possibility that these subjects changed their food pattern toward a higher consumption of high-fat foods and a lower consumption of fish. 
Table 2. Age-Adjusted Mean Daily Intake of Selected Nutrients and Fish According to Incidence of Dementia:

The Rotterdam Study

\begin{tabular}{lccc} 
& \multicolumn{2}{c}{ Dementia at Follow-Up } & Difference $(95 \% \mathrm{CI})$ \\
\cline { 2 - 4 } Daily Intake & No $(\mathrm{n}=5,328)$ & Yes $(\mathrm{n}=58)$ & $509(-40,1,058)$ \\
\hline Total energy $(\mathrm{kJ})$ & $8,254(28.8)^{\mathrm{a}}$ & $8,763(278.5)$ & $1.7(0.1,3.3)$ \\
Total fat (en\%) & $36.5(0.08)$ & $38.2(0.82)$ & $1.1(0.3,1.9)$ \\
Saturated fat (en\%) & $14.4(0.04)$ & $15.5(0.42)$ & $1.6(-0.53,3.44)$ \\
Cholesterol (mg/MJ) & $28.5(0.10)$ & $30.1(0.99)$ & $-0.4(-1.2,0.3)$ \\
Linoleic acid (en\%) & $5.7(0.04)$ & $5.3(0.38)$ & $-4.8(-9.7,0.1)$ \\
Fish $(\mathrm{g})$ & $15.8(0.26)$ & $10.9(2.48)$ & \\
\hline
\end{tabular}

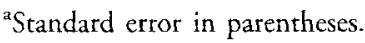

$\mathrm{CI}=$ confidence interval; en $\%=$ intake as percentage of total energy intake.

Table 3. Risk of Dementia According to Tertiles of Energy-Adjusted Fat Intake Estimated by Multiple Logistic Regression: The Rotterdam Study

\begin{tabular}{|c|c|c|c|c|c|c|c|}
\hline & \multicolumn{3}{|c|}{ Total Dementia $(\mathrm{n}=58)$} & \multicolumn{2}{|c|}{$\begin{array}{c}\text { Alzheimer's Disease Without } \\
\text { Cerebrovascular Disease } \\
(\mathrm{n}=37)\end{array}$} & \multicolumn{2}{|c|}{$\begin{array}{l}\text { Dementia with a } \\
\text { Vascular Component } \\
\qquad(\mathrm{n}=12)\end{array}$} \\
\hline & $\mathrm{n}$ & $R R(95 \% \mathrm{CI})^{\mathrm{b}}$ & $p$ Trend & $R R(95 \% \mathrm{CI})^{\mathrm{b}}$ & $p$ Trend & $R R(95 \% \mathrm{CI})^{\mathrm{b}}$ & $p$ Trend \\
\hline \multicolumn{8}{|l|}{ Total fat (g/day) } \\
\hline$\leq 75.5$ & 1,795 & $1.0(\mathrm{ref})$ & & 1.0 (ref) & & $1.0($ ref $)$ & \\
\hline $75.5-85.5$ & 1,768 & $2.1(0.9-4.7)$ & & $1.8(0.7-4.5)$ & & $1.7(0.3-10.3)$ & \\
\hline$>85.5$ & 1,823 & $2.4(1.1-5.2)$ & 0.02 & $1.6(0.6-3.9)$ & 0.25 & $3.0(0.6-14.7)$ & 0.02 \\
\hline \multicolumn{8}{|c|}{ Saturated fat ( $\mathrm{g} /$ day) } \\
\hline$\leq 29.0$ & 1,873 & 1.0 (ref) & & 1.0 (ref) & & 1.0 (ref) & \\
\hline $29.0-34.0$ & 1,653 & $1.8(0.8-4.1)$ & & $1.8(0.7-4.7)$ & & $1.0(0.1-7.2)$ & \\
\hline$>34.0$ & 1,860 & $1.9(0.9-4.0)$ & 0.12 & $1.3(0.5-3.3)$ & 0.66 & $2.9(0.6-13.8)$ & 0.01 \\
\hline \multicolumn{8}{|c|}{ Cholesterol (mg/day) } \\
\hline$\leq 208.5$ & 1,808 & $1.0(\mathrm{ref})$ & & 1.0 (ref) & & 1.0 (ref) & \\
\hline $208.5-254.5$ & 1,785 & $1.1(0.5-2.4)$ & & $1.2(0.5-3.0)$ & & $1.1(0.3-4.6)$ & \\
\hline$>254.5$ & 1,793 & $1.7(0.9-3.2)$ & 0.11 & $1.3(0.6-3.1)$ & 0.40 & $0.9(0.2-3.7)$ & 0.80 \\
\hline
\end{tabular}

ancludes vascular dementia and Alzheimer's disease with cerebrovascular disease; other dementias are excluded from these analyses.

${ }^{\mathrm{b}}$ Adjusted for age, sex, education, and total energy intake.

$R R=$ relative risk; $\mathrm{CI}=$ confidence interval; ref $=$ reference category.

Table 4. Risk of Dementia According to Tertiles of Energy-Adjusted Linoleic Acid and Fish Intake Estimated by Multiple Logistic Regression: The Rotterdam Study

\begin{tabular}{|c|c|c|c|c|c|c|c|}
\hline & \multicolumn{3}{|c|}{ Total Dementia $(\mathrm{n}=58)$} & \multicolumn{2}{|c|}{$\begin{array}{l}\text { Alzheimer's Disease Without } \\
\text { Cerebrovascular Disease } \\
(\mathrm{n}=37)\end{array}$} & \multicolumn{2}{|c|}{$\begin{array}{l}\text { Dementia with a } \\
\text { Vascular Component } \\
\qquad(\mathrm{n}=12)\end{array}$} \\
\hline & n & $R R(95 \% \mathrm{CI})^{\mathrm{b}}$ & $p$ Trend & $R R(95 \% \mathrm{CI})^{\mathrm{b}}$ & $p$ Trend & $R R(95 \% \mathrm{CI})^{\mathrm{b}}$ & $p$ Trend \\
\hline \multicolumn{8}{|c|}{ Linoleic acid (g/day) } \\
\hline$\leq 9.5$ & 1,871 & 1.0 (ref) & & $1.0(\mathrm{ref})$ & & $1.0(\mathrm{ref})$ & \\
\hline $9.5-15.0$ & 1,775 & $1.2(0.7-2.3)$ & & $0.9(0.4-1.9)$ & & $1.0(0.3-3.6)$ & \\
\hline$>15.0$ & 1,740 & $0.6(0.3-1.2)$ & 0.23 & $0.7(0.3-1.5)$ & 0.17 & $0.4(0.1-1.9)$ & 0.09 \\
\hline \multicolumn{8}{|l|}{ Fish (g/day) } \\
\hline$\leq 3.0$ & 1,807 & 1.0 (ref) & & 1.0 (ref) & & 1.0 (ref) & \\
\hline $3.0-18.5$ & 1,773 & $0.8(0.4-1.4)$ & & $0.9(0.4-1.8)$ & & $0.6(0.2-2.5)$ & \\
\hline$>18.5$ & 1,806 & $0.4(0.2-0.9)$ & 0.03 & $0.3(0.1-0.9)$ & 0.005 & $0.7(0.2-2.8)$ & 0.39 \\
\hline
\end{tabular}

${ }^{a}$ Includes vascular dementia and Alzheimer's disease with cerebrovascular disease; other dementias are excluded from these analyses.

${ }^{b}$ Adjusted for age, sex, education, and total energy intake.

$R R=$ relative risk; $\mathrm{CI}=$ confidence interval; ref $=$ reference category. 
Finally, persons with prevalent cardiovascular disease may have altered their diet toward a lower intake of saturated fat and cholesterol. Because they might also have a higher risk of dementia, this could have resulted in an underestimation of our estimates. However, the association between saturated fat or cholesterol and dementia did not change when we excluded subjects with cardiovascular disease or subjects using a prescribed diet.

We found a borderline significant association of saturated fat and cholesterol intake with incident dementia. This association may have been mediated by cardiovascular disease. A high intake of saturated fat and cholesterol has been consistently associated with an increased risk of coronary heart disease $[4,5]$, and there are some data showing an association between dietary saturated fat and cerebrovascular disease [6]. Cardiovascular disease has subsequently been related to dementia, especially vascular dementia [7-9]. The hypothesis of a vascular mechanism is consistent with the finding that a high saturated fat intake was most strongly related to dementia with a vascular component. Therefore, we expected that adjustment for cardiovascular disease would have attenuated our estimates, but it did not. The reason for this may be that we adjusted for cardiovascular disease at baseline, whereas it would have been optimal to adjust for cardiovascular disease that developed during follow-up and before onset of dementia. Furthermore, only few subjects suffered from clinical stroke [25]. Silent cerebrovascular disease, which may be an important intermediate as well, is thought to be at least four times more frequent than clinically recognized strokes [29]. We had information on indicators of atherosclerosis, but only in a relatively small subset of subjects, and adjustment for them did not change the results either. This may have been due to measurement error, lack of power, or the fact that these indicators of atherosclerosis might not correlate exactly with subclinical lesions of the brain.

The relation of a high-fat diet to dementia may also be related to other factors associated with socioeconomic status, such as lower education, smoking, and alcohol consumption. Adjustment for these factors did not change our results, but we cannot exclude residual confounding. There may be alternate mechanisms; for instance, cholesterol may have a more direct effect on the brain. An experimental study in rabbits showed that $\beta$-amyloid accumulated in a dose-dependent way in brains of rabbits fed a high-cholesterol diet [30].

In the present study, a high linoleic acid intake was slightly, but not significantly, associated with a lower risk of dementia. Although some studies have suggested that linoleic acid may be atherogenic by increasing the oxidative modification of low-density lipoprotein cholesterol [31], our result is compatible with a beneficial effect of linoleic acid on the lipid profile [12].
Fish, as a marker of n-3 PUFAs in fish, was associated with a reduced risk of dementia, primarily of $A D$, even at relatively low levels of fish consumption. The results of the present study are similar to another study, in which we found an inverse association between a comparable fish consumption and the risk of cognitive impairment and decline [32]. A crosssectional study in Norway indicated that increased plasma phospholipid levels of n-3 PUFAs were observed with a usual intake of one to two servings of fish per week [33]. There are several biologically plausible mechanisms for the association between fish and $\mathrm{AD}$. First, fish may protect against dementia by reducing the risk of cardiovascular disease [34, 35]. Among subjects with neuropathological brain lesions typical for $\mathrm{AD}$, brain infarcts, and especially lacunar infarcts, more often resulted in clinical dementia [10]. In addition, results from the Rotterdam Study indicate that atherosclerosis is related to an increased risk of $\mathrm{AD}$ [9]. Our finding of a particular association with pure $\mathrm{AD}$ may suggest a different mechanism as well. The $n-3$ PUFAs in fish have antiinflammatory properties; that is, they can decrease the production of proinflammatory cytokines in humans [36]. Immune processes are believed to be involved in the pathogenesis of AD. Increased concentrations of acute phase reactants and other markers of immune processes, such as cytokines, have been found in brain tissue of patients with $\mathrm{AD}$ [37]. In addition, interleukin-1 may regulate the amyloid precursor protein synthesis, resulting in amyloid deposits [38]. Epidemiological studies have suggested that the use of nonsteroidal antiinflammatory drugs reduced the risk of $A D$ [39]. Finally, n-3 PUFAs also play a role in brain development and the functioning of nerve membranes [40], and they can still be incorporated in the brains of animals after the period of brain development [41]. They may therefore be of importance in the regeneration of nerve cells [42]. A human autopsy study showed that the PUFA content, including the n-3 PUFA docosahexaenoic acid, was decreased in the hippocampus and frontal gray matter of $\mathrm{AD}$ brains, whereas the fatty acid composition of normal aged brains was not altered [43].

In conclusion, this study suggests that a high saturated fat and cholesterol intake raises the risk of dementia, in particular of dementia with a vascular component. In addition, fish consumption may reduce the risk of dementia, and primarily of $\mathrm{AD}$. The associations we report here are biologically plausible. However, because this is the first prospective study examining the relation of dietary fat intake to the risk of dementia, additional epidemiological studies with a longer follow-up period are needed to address this issue. If these findings are replicated, they could have important implications for reducing the risk of dementia. 
This study was supported by grants from the NESTOR stimulation program for geriatric research (supported by The Netherlands Ministries of Health and Education), The Netherlands Heart Foundation, The Netherlands Organisation for Scientific Research (NWO), The Netherlands Prevention Fund, and the municipality of Rotterdam.

We gratefully acknowledge Dr F. van Harskamp, the dieticians, the co-workers, and the cooperating general practitioners in Ommoord; and Dr E. J. M. Feskens for her critical review of the manuscript.

\section{References}

1. Giem P, Beeson WL, Fraser GE. The incidence of dementia and intake of animal products: preliminary findings from the adventist health study. Neuroepidemiology 1993;12:28-36

2. Burns A, Marsh A, Bender DA. Dietary intake and clinical, anthropometric and biochemical indices of malnutrition in elderly demented patients and non-demented subjects. Psychol Med 1989;19:383-391

3. Broe GA, Henderson AS, Creasey $\mathrm{H}$, et al. A case-control study of Alzheimer's disease in Australia. Neurology 1990;40:16981707

4. McGee DL, Reed DM, Yano K, et al. Ten-year incidence of coronary heart disease in the Honolulu Heart Program. Relationship to nutrient intake. Am J Epidemiol 1984;119:667676

5. Shekelle RB, Shryock AM, Oglesby P, et al. Diet, serum cholesterol, and death from coronary heart disease. The Western Electric study. N Engl J Med 1981;304:65-70

6. Sasaki S, Zhang $\mathrm{X}-\mathrm{H}$, Kesteloot $\mathrm{H}$. Dietary sodium, potassium, saturated fat, alcohol, and stroke mortality. Stroke 1995;26: 783-789

7. Awad IA, Spetzler RF, Hodak JA, et al. Incidental subcortical lesions identified on magnetic resonance imaging in the elderly. I. Correlation with age and cerebrovascular risk factors. Stroke 1986;17:1084-1089

8. Erkinjuntti T, Hachinski VC. Rethinking vascular dementia. Cerebrovasc Dis 1993;3:3-23

9. Hofman A, Ott A, Breteler MMB, et al. Atherosclerosis, apolipoprotein $\mathrm{E}$ and the prevalence of dementia and Alzheimer's disease in the Rotterdam study. Lancet 1997;349:151-154

10. Snowdon DA, Greiner LH, Mortimer JA, et al. Brain infarction and the clinical expression of Alzheimer's disease. The Nun study. JAMA 1997;277:813-817

11. Kinsella JE, Lokesh B, Stone RA. Dietary n-3 polyunsaturated fatty acids and amelioration of cardiovascular disease: possible mechanisms. Am J Clin Nutr 1990;52:1-28

12. Mensink RP, Katan MB. Effect of dietary fatty acids on serum lipids and lipoproteins. A meta-analysis of 27 trials. Arterioscler Thromb 1992;12:911-919

13. Wood DA, Riemersma RA, Butler $S$, et al. Linoleic and eicosapentaenoic acids in adipose tissue and platelets and risk of coronary heart disease. Lancet 1987;1:177-182

14. Hofman A, Grobbee DE, de Jong PTVM, van den Ouweland FA. Determinants of disease and disability in the elderly: the Rotterdam Elderly study. Eur J Epidemiol 1991;7:403--422

15. Roth M, Tym E, Mountjoy CQ, et al. CAMDEX: a standardized instrument for the diagnosis of mental disorders in the elderly with special reference to the early detection of dementia. Br J Psychiatr 1986;149:698--709

16. Goldbohm RA, van den Brandt PA, Brants HAM, et al. Validation of a dietary questionnaire used in a large-scale prospective cohort study on diet and cancer. Eur J Clin Nutr 1994; $48: 253-265$
17. Willett WC, Sampson L, Stampfer MJ, et al. Reproducibility and validity of a semiquantitative food frequency questionnaire. Am J Epidemiol 1985;122:51-65

18. Stichting Nederlands Voedingsstoffenbestand. NEVO Tabel 1993. Den Haag, 1993

19. Ott A, Breteler MMB, van Harskamp F, et al. Prevalence of Alzheimer's disease and vascular dementia: association with education. The Rotrerdam study. Br Med J 1995;310:970-973

20. Folstein MF, Folstein SE, McHugh PR. "Mini-Mental State." A practical method for grading the cognitive state of patients for the clinician. J Psychiatr Res 1975;12:189-198

21. Copeland JRM, Kelleher MJ, Kellett JM, et al. A semistructured clinical interview for the assessment of diagnosis and mental state in the elderly: the Geriatric Mental State Schedule. I. Development and reliability. Psychol Med 1976; $6: 439-449$

22. McKhann G, Drachman D, Folstein M, et al. Clinical diagnosis of Alzheimer's disease: report of the NINCDS-ADRDA Work Group under the auspices of Department of Health and Human Services Task Force on Alzheimer's Disease. Neurology 1984;34:939-944

23. Román GC, Tatemichi TK, Erkinjuntti $T$, et al. Vascular dementia: diagnostic criteria for research studies. Report of the NINDS-AIREN International Workshop. Neurology 1993;43: $250-260$

24. American Psychiatric Association. Diagnostic and statistical manual of mental disorders. 3rd ed, revised. American Psychiatric Association, Washington, DC: 1987

25. Bots ML, Looman SJ, Koudstaal PJ, et al. Prevalence of stroke in the general population. The Rotterdam Study. Stroke 1996; 27:1499-1501

26. Bots ML, van Swieten JC, Breteler MMB, et al. Cerebral white matter lesions and atherosclerosis in the Rotterdam study. Lancet 1993;341:1232-1237

27. Willett WC, Stampfer MJ. Total energy intake: implications for epidemiologic analyses. Am J Epidemiol 1986;124:17-27

28. van Stavern WA, de Groot LCPGM, Blauw YH, van der Wielen RPJ. Assessing diets of elderly people: problems and approaches. Am J Clin Nutr 1994;59(suppl):221S-223S

29. White $\mathrm{L}$. Is silent cerebrovascular disease an important cause of late-life cognitive decline? J Am Geriatr Soc 1996;44:328330

30. Sparks DL, Scheff SW, Hunsaker JC III, et al. Induction of Alzheimer-like $\beta$-amyloid immunoreactivity in the brains of rabbits with dietary cholesterol. Exp Neurol 1994;126:88-94

31. Reaven PD, Grasse BJ, Tribble DL. Effects of linoleateenriched and oleate-enriched diets in combination with alphatocopherol on the susceptibility of LDL and LDL subfractions to oxidative modification in humans. Arterioscler Thromb 1994; $14: 557-566$

32. Kalmijn S, Feskens EJM, Launer LJ, Kromhout D. Polyunsaturated fatty acids, antioxidants and cognitive function in very old men. Am J Epidemiol 1997;145:33-41

33. Bønaa KH, Bjerve KS, Nordøy A. Habitual fish consumption, plasma phospholipid fatty acids, and serum lipids: the Tromsø study. Am J Clin Nutr 1992;55:1126-1134

34. Keli SO, Feskens EJM, Kromhout D. Fish consumption and risk of stroke. The Zutphen study. Stroke 1994:25:328-332

35. Kromhout $\mathrm{D}$, Bosschieter $\mathrm{EB}$, de Lezenne $\mathrm{C}$. The inverse relation between fish consumption and 20-year mortality from coronary heart disease. N Engl J Med 1985;312:1205-1209

36. Blok WL, Katan MB, van der Meer JWM. Modulation of inflammation and cytokine production by dietary ( $n-3)$ fatty acids. J Nutr 1996;126:1515-1533

37. Griffin WST, Stanley LC, Ling C, et al. Brain interleukin 1 
and S-100 immunoreactivity are elevated in Down syndrome and Alzheimer disease. Proc Natl Acad Sci USA 1989;86:76117615

38. Vandenabecle P, Fiers W. Is amyloidogenesis during Alzheimer's disease due to an IL-1-/IL-6-mediated "acute phase response" in the brain? Immunol Today 1991;12:217-219

39. Andersen K, Launer LJ, Ott A, et al. Do nonsteroidal antiinflammatory drugs decrease the risk for Alzheimer's disease? The Rotterdam study. Neurology 1995;45:1441-1445

40. Bourre J-M, Ftancois M, Youyou A, et al. The effects of dietary $\alpha$-linoleic acid on the composition of nerve membranes, enzy- matic activity, amplitude of electrophysiological parameters, resistance to poisons and performance of learning tasks in rats. J Nutr 1989;119:1880-1892

41. Neuringer $M$, Connor WE. $n-3$ fatty acids in the brain and retina: evidence for their essentiality. Nutr Rev 1986;44:285294

42. Compston A. Brain repair: an overview. J Neurol 1994;241: S1-S4

43. Söderberg M, Edlund C, Kristensson K, Dallner G. Fatty acid composition of brain phospholipids in aging and in Alzheimer's disease. Lipids 1991;26:421-425 